Proceedings

\title{
Influence of Ball Impact Location on Racquet Kinematics, Forearm Muscle Activation and Shot Accuracy during the Forehand Groundstrokes in Tennis ${ }^{+}$
}

\author{
Masahiro Ikenaga ${ }^{1, *}$, Nobue Okuma ${ }^{1}$, Hiroki Nishiyama ${ }^{2}$, Shinichiro Chiba ${ }^{1}$, \\ Katsutoshi Nishino ${ }^{3}$, Go Omori ${ }^{4}$ and Hiroyuki Nunome ${ }^{5}$ \\ 1 Advanced Research and Development, YONEX CO., LTD, 900-1 Tsukanoyama, Nagaoka-city, \\ Niigata 949-5123, Japan; n-okuma@yonex.co.jp (N.O.); shinichiro_chiba@yonex.co.jp (S.C.) \\ 2 Technology Development Department, YONEX CO., LTD, 900-1 Tsukanoyama, Nagaoka-city, \\ Niigata 949-5123, Japan; h-nishiyama@yonex.co.jp \\ 3 Niigata Institute for Health and Sports Medicine, 67-12 Seigoromachi Chuo-ku, Niigata-city, \\ Niigata 950-0933, Japan; k.nishino@ken-supo.jp \\ 4 Faculty of Health and Science, Niigata University of Health and Welfare, 1398 Shimami-cho, Kita-ku, \\ Niigata-city, Niigata 950-3198, Japan; omori@nuhw.ac.jp \\ 5 Faculty of Sports and Health Science, Fukuoka University, 8-19-1 Nanakuma, Jonan-ku, Fukuoka-city, \\ Fukuoka 814-0180, Japan; nunome@fukuoka-u.ac.jp \\ * Correspondence: m-ikenaga@yonex.co.jp; Tel.: +81-0258-94-2121 \\ + Presented at the 13th Conference of the International Sports Engineering Association, Online, \\ 22-26 June 2020.
}

Published: 15 June 2020

\begin{abstract}
We aimed to clarify the effect of ball-racquet impact locations on the dynamic behavior of tennis racquet, the accuracy of shots and muscle activation of the forearm. Eight male intermediate tennis players performed ten forehand groundstrokes. A motion capture system was used to measure the motions of racquet, ball and human body at $2000 \mathrm{~Hz}$, and electromyography (EMG) activities of wrist extensor and flexor muscles were measured simultaneously. The flight parameters of the ball were measured by ballistic measurement equipment. All shots were divided into tertiles based on ball impact location along the lateral axis of tennis racquet. We found that the off-center, upper-side impact induces a larger muscular activity in extensor carpi radialis. Passive radial deviation of the wrist occurring immediately after ball impact may account for this. Furthermore, the off-center, upper-side impact could be associated with a missed shot having a lower, outward ball launch angle.
\end{abstract}

Keywords: tennis elbow; off-center impacts; athletic performance

\section{Introduction}

The main advancement in recent tennis racquets is that they have highly flexible shape design while maintaining lightweight, high-rigidity and high-strength by combining various composite materials such as carbon fiber reinforced plastic (CFRP). In particular, the size of the lowest vibration area (sweet spot) is one of the most important target elements for the tennis racquet. It has been known that the magnitudes of shock and vibration transmitted to the arm is influenced by the offcenter ball impact location [1-3]. A tennis racquet with a larger sweet spot, therefore, would attenuate shock force transmitted to the arm and reduce the number of missed shots due to racquet variations. A previous study which combined electromyography (EMG) of the forearm muscles and ball impact 
videography revealed that players with lateral epicondylitis (LE) showed greater EMG for wrist extensors and pronator muscles [4]. Moreover, players with LE consistently hit the ball at off-center locations, which typically caused the upward tilting of the racquet face after ball impact. The onset risk of LE is also related to the level of tennis skill, as previous studies has suggested that unskilled players often grip the racquet forcefully [5], and tighter racquet grip causes an increase in wrist joint torque during racquet rotation due to off-center hits [2]. However, the interactions among the location of ball impact on a racquet; the behavior of the racquet just before, during and just after ball impact; and forearm muscle activity during forehand groundstrokes, have never been quantified systematically.

The purpose of this study was to clarify: (1) the effect of the racquet behavior on the forearm muscle activity due to different ball impact location during the forehand groundstrokes; and, (2) the relationship between ball impact location and shot accuracy using high-speed 3D motion analysis. We hypothesized that the upward tilting of a racquet caused by off-center, upper-side hitting during forehand groundstrokes would increase wrist extensor muscle activity due to eccentric contraction, raise ball launch angle and decreasing ball spin rate.

\section{Methods}

\subsection{Subjects}

Eight male intermediate tennis players (age: $29.5 \pm 5.5$ years; height: $1.74 \pm 0.04 \mathrm{~m}$; weight: $63.6 \pm$ $5.9 \mathrm{~kg}$; tennis practice: $14.3 \pm 4.4$ years) were recruited for the study. Seven subjects were right-handed and one was left-handed. The Ethics Committee on Human Research of the Niigata University of Health and Welfare approved this study, and all subjects gave their consent.

\subsection{Experimental Desighn and Data Sampling}

The subjects used YONEX VCORE100 racquets with two different weights (head size: 100"; length: 27"; weight: 280 or $300 \mathrm{~g}$; string pattern: $16 \times 19$ ) strung with nylon (PTGP 125; YONEX Co., Ltd., Tokyo, Japan) at $50 \mathrm{lbs}$ of tension. They were asked to choose the grip size and the weight from two types, respectively, that felt most comfortable to them. The experiment was conducted in a biomechanics laboratory that mimics half of a tennis court (Figure 1a). After warm-up, the subjects performed 10 straight forehand groundstrokes on balls emitted from a ball machine (Tennis Tutor plus; Sports Tutor Inc., Burbank, CA, USA) at a speed of $12 \mathrm{~m} / \mathrm{s}$ towards a target (center of horizontal line drawn $1.6 \mathrm{~m}$ high) that was $13 \mathrm{~m}$ from the subject. The emitted balls were bounced once on the floor and adjusted to a height of approximately $1 \mathrm{~m}$ in the subject's hitting area. Subjects were instructed to hit the forehand at the same intensity as their best control shot in a competitive tennis match.

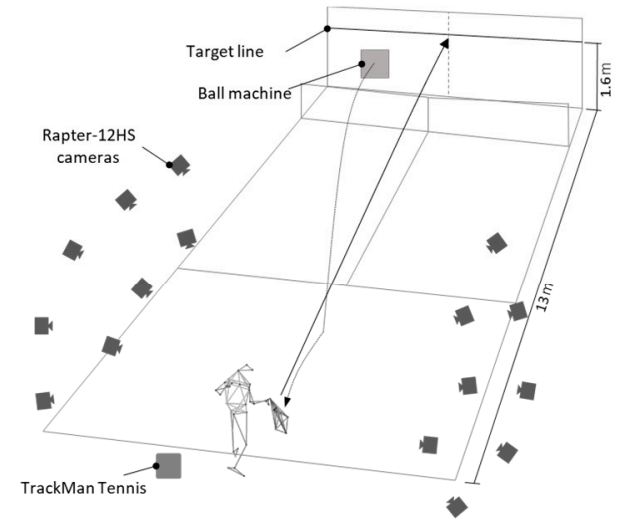

(a)

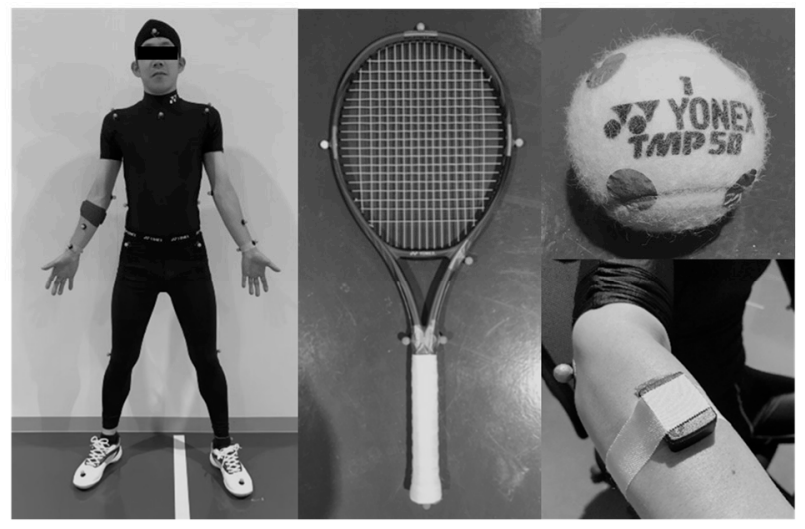

(b)

Figure 1. (a) The experimental set-up; (b) Placement of the reflective markers in the body, the racquet and the ball. Two pairs of surface electromyography (EMG) were placed at the extensor carpi radialis muscle and flexor carpi radialis muscle. 
Three-dimensional coordinates were measured using a high-resolution motion-capture system (Rapter-12HS; Motion Analysis Corporation, Rohnert Park, CA, USA) consisting of 16 Rapter-12HS cameras. A sampling rate of $2000 \mathrm{~Hz}$ and a shutter speed of $2000 \mathrm{~Hz}$ were used. Thirty-five retroreflective markers were placed on the subject's body, 7 markers were fixed on the racquet, and double-sided 8 circular retro-reflective stickers with a diameter of $16 \mathrm{~mm}$ were attached to the balls (Figure 1b). Surface EMG wireless sensors (Trigno Avanti; Delsys Inc., Natick, MA, USA) were placed on the extensor carpi radialis muscle (ECR) and the flexor carpi radialis muscle (FCR) of the subject's dominant arm. EMG recording was sampled at $2000 \mathrm{~Hz}$. The three-dimensional coordinates and EMG were synchronized using software (Cortex 7.1.2.1822; Motion Analysis Corporation, Rohnert Park, CA, USA). To measure ball flight parameters, TrackMan tennis (TrackMan AS, Vedbaek, Denmark) was used. We evaluated ball velocity, spin rate, launch angle and ball placement on the net.

\subsection{Data Analysis}

Three-dimensional coordinates were expressed as a right-handed coordinate system in which each axis was orthogonalized and normalized. A custom written program (MATLAB; MathWorks Inc., Natick, MA, USA) was used to calculate joint angle as previously described [6]. We quantified four explanatory variables of racquet behavior: racquet head velocity, racquet trajectory and racquetface angle at $3 \mathrm{~ms}$ after the instant of the ball impact. Racquet trajectory and the angle from the horizontal were calculated from the horizontal and vertical velocity of the racquet head marker at impact. The racquet-face angle was determined as the angle between the vector normal to the racquet surface (defined by three markers attached on the racquet) and the vertical axis. For calculating impact location, the geometric center of the ball was estimated from the four ball markers by a leastsquares technique, assuming each marker was positioned the same distance from the ball center. The ball center position was converted to the racquet local coordinate system and calculated relative to the center of the racquet stringbed.

The motion of forehand groundstrokes from the start swing (SS) to the end of follow through was divided into four phases (phase I, early acceleration; phase II, late acceleration; phase III, early follow-through; phase IV, late follow-through [4]) based on the following five time events (Figure 2). SS was determined as the instant when players started trunk rotation toward the ball. The instant of the maximal shoulder joint external rotation was determined as MER. The moment of ball impact (IMP) was defined as the one frame before when the racquet apparently contacted with the ball. After a sudden deceleration of the racquet head due to ball impact, the racquet head was reaccelerated for follow-through. The instant when the maximal racquet head velocity re-increased was determined as MVR. Threefold the time between IMP to MVR was determined as the finish (FIN). The period of IMP was normalized $100 \%$, and FIN was normalized $150 \%$.

The average EMG during each phase were used for data analysis. The average normalized values of EMG for each muscle were expressed as a percentage of the maximum EMG level recorded during all the swings by each subject.

\subsection{Statistical Analysis}

All shots were divided into tertiles based on ball impact locations along the lateral axis of racquet: below, center and above. A total of 77 shots were chosen for further analysis, excluding a few trials in which ball impact location was not feasible to detect due to a smaller number of ball markers $(<4)$ captured appropriately. We classified these trials into tertiles: the off-center impact below (Below $\mathrm{N}=25)$, the center impact (Center $\mathrm{N}=26$ ), and the off-center impact above (Above $\mathrm{N}=$ 26). The results were given as means \pm SD. A one-way analysis of variance (ANOVA) with Bonferroni post-hoc pair-wise comparisons were used for the assessment of the associations between the impact location and parameters of racquet behavior, EMG and ball flight behavior. Significance was set at $p<0.05$. 


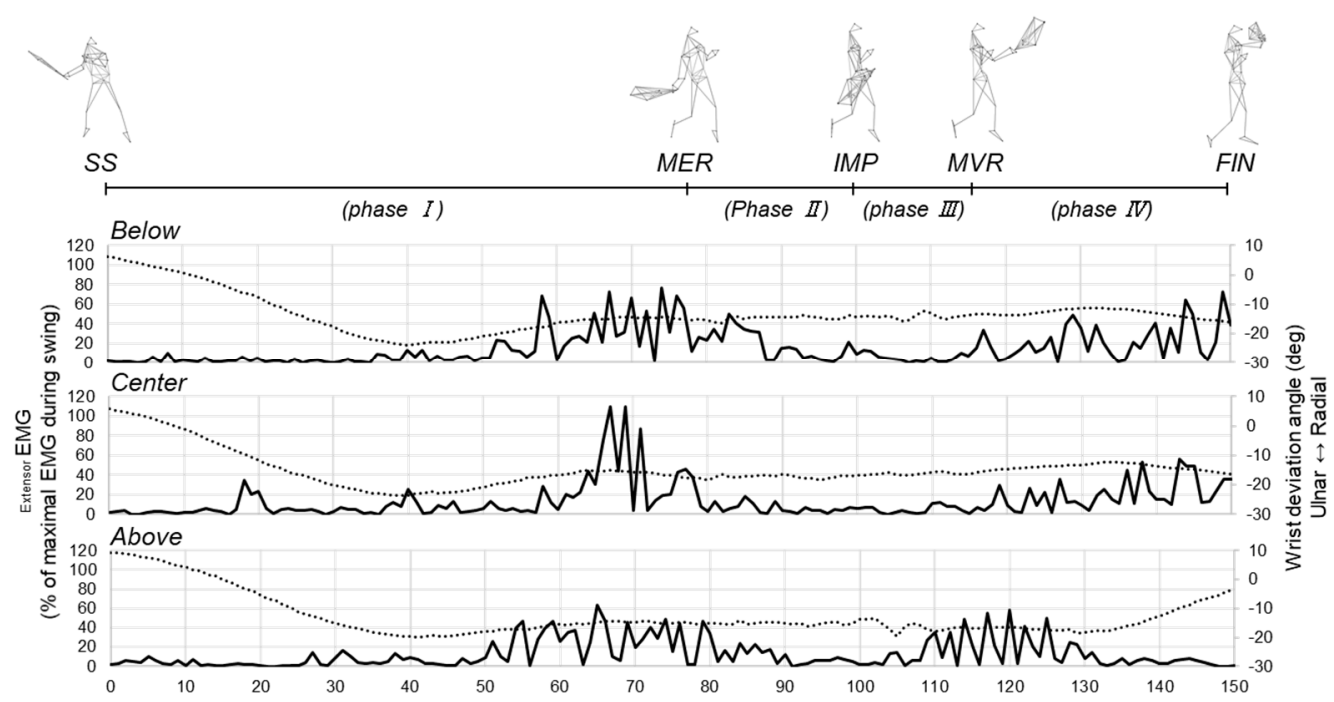

Figure 2. Phases of swing motion, changes in wrist extensor electromyography (EMG; solid line) and wrist ulnar/radial deviation angle (dotted line) in three impact location patterns categorized below, above or on (Center) the longitudinal axis. Data were from one selected subject. SS, start swing; MER, maximal shoulder external rotation; IMP, ball impact; MVR, maximal velocity during recovery phase; FIN, finish swing.

\section{Results}

Table 1 presents the comparison of each parameter among three impact location conditions. The average (range) of impact location from the longitudinal axis of the racquet of each condition were as follows: Below, $-12.8 \mathrm{~mm}(-37.3 \mathrm{~mm}$ to $-1.1 \mathrm{~mm})$; Center, $6.2 \mathrm{~mm}$ (-0.9 mm to $13.1 \mathrm{~mm})$; Above, $33.7 \mathrm{~mm}$ (13.5 $\mathrm{mm}$ to $92.4 \mathrm{~mm}$ ). No significant difference was observed for the racquet head velocity and racquet trajectory among the three conditions. The racquet-face impact angle in the Above condition was significantly lower (close racquet-face) than that of the Center $(p<0.01)$. In the Above condition, the racquet-face angle opened a few degrees immediately after ball impact.

The EMG normalized activities for the ECR during phase III were significantly larger than those in the Above condition than in the Center condition $(p<0.05$, Figure 3a). No significant difference was observed in EMG activities for the ECR between the Below condition and the Center condition. A greater normalized EMG activity for the FCR during phase IV was observed in the Below condition than in the Center condition $(p<0.05$, Figure 3b).

Figure $3 \mathrm{c}$ shows ball passing position on a net. The Above condition had a lower height and passed right when compared to the Center condition and the Below condition; however, the difference was not statistically significant. No significant differences were observed for ball velocity and spin rate among the three conditions (Table 1). The launch angle was significantly lower in the Above condition than those of the Center condition and the Below condition $(p<0.05)$.

(a)

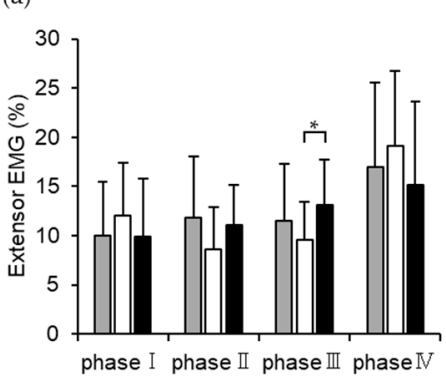

(b)

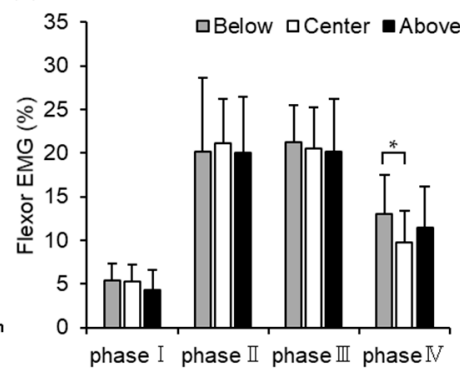

(c)

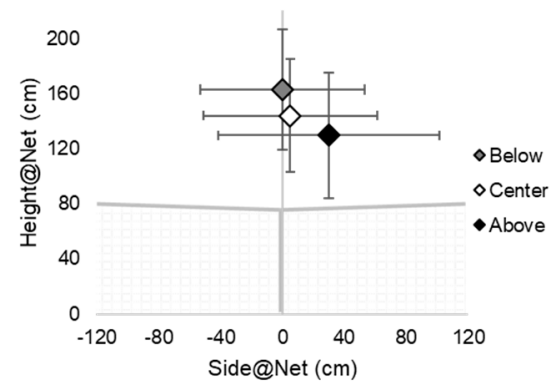

Figure 3. Average EMG for the extensor carpi radius muscle (ECR) (a) and the flexor carpi radialis muscle (FCR) (b) at each phase three impact location patterns. (c) Ball passing position on the net. * Indicates significant difference $(p<0.05)$ between impact location conditions. 
Table 1. Summarized results.

\begin{tabular}{|c|c|c|c|c|c|c|}
\hline \multirow[b]{2}{*}{ Impact location } & \multicolumn{2}{|c|}{$\begin{array}{c}\text { Below } \\
(\mathrm{N}=25)\end{array}$} & \multicolumn{2}{|c|}{$\begin{array}{c}\text { Center } \\
(\mathrm{N}=26)\end{array}$} & \multicolumn{2}{|c|}{$\begin{array}{c}\text { Above } \\
(\mathrm{N}=26)\end{array}$} \\
\hline & & & & & & 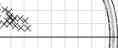 \\
\hline & Mean & (SD) & Mean & (SD) & Mean & (SD) \\
\hline \multicolumn{7}{|l|}{ Racquet behaviors } \\
\hline Head velocity $(\mathrm{km} / \mathrm{h})$ & 127.1 & $(9.1)$ & 124.2 & (9.7) & 128.4 & $(7.9)$ \\
\hline Racquet trajectory (deg) & 18.0 & $(5.6)$ & 16.5 & $(4.3)$ & 18.6 & $(5.5)$ \\
\hline Racquet-face impact angle (deg) & 85.6 & $(2.9)$ & 85.5 & $(2.3)$ & 82.8 & $(4.1)^{* *}$ \\
\hline Racquet rotation (deg) & -1.1 & $(1.7)$ & 0.8 & (1.6) & 3.0 & $(1.7)^{* *}$ \\
\hline \multicolumn{7}{|l|}{ Ball trajectory } \\
\hline Ball velocity $(\mathrm{km} / \mathrm{h})$ & 134.8 & $(10.7)$ & 136.0 & $(8.4)$ & 135.0 & $(10.8)$ \\
\hline Launch angle (deg) & 6.8 & $(2.7)$ & 5.4 & $(2.1)$ & 4.9 & $(1.6)^{+}$ \\
\hline Spin rate $(\mathrm{rpm})$ & 1696 & (459) & 1490 & (322) & 1736 & $(506)$ \\
\hline Side@NET (cm) & 0.1 & $(52.9)$ & 5.1 & $(56.2)$ & 30.0 & (71.2) \\
\hline Height@NET (cm) & 162.9 & $(43.4)$ & 144.0 & (41.0) & 129.6 & $(45.5)$ \\
\hline \multicolumn{7}{|l|}{ Normalized wrist EMG } \\
\hline \multirow[t]{4}{*}{ Extensor EMG (\%) } & 10.0 & $(5.6)$ & 12.0 & $(5.4)$ & 9.9 & $(5.8)$ \\
\hline & 11.8 & (6.3) & 8.6 & $(4.3)$ & 11.1 & $(4.1)$ \\
\hline & 11.5 & (5.7) & 9.6 & (3.9) & 13.1 & $(4.6) *$ \\
\hline & 17.0 & (8.6) & 19.1 & (7.6) & 15.1 & $(8.4)$ \\
\hline \multirow[t]{4}{*}{ Flexor EMG (\%) } & 5.4 & (1.9) & 5.3 & (1.9) & 4.3 & (2.3) \\
\hline & 20.2 & (8.4) & 21.1 & (5.1) & 20.0 & (6.4) \\
\hline & 21.2 & $(4.3)$ & 20.5 & $(4.7)$ & 20.2 & (6.1) \\
\hline & 13.0 & $(4.5)$ & 9.7 & $(3.7)$ & 11.4 & $(4.7)$ \\
\hline
\end{tabular}

SD, standard deviation; EMG, electromyography. ${ }^{*} p<0.05,{ }^{* *} p<0.01$, Significant difference with the

Center condition. ${ }^{\dagger} p<0.05$, Significant difference with the Below condition.

\section{Discussion}

In this study, we hypothesized that a ball hitting at off-center, upper-side of a tennis racquet would increase the wrist extensor muscle activity, decrease the ball spin rate and raise the ball launch angle due to upward tilted racquet face after ball impact. In contrast to our hypothesis, the launch angle of the ball in off-center, upper-side impact condition (Above) was significantly lower than the other impact conditions and the ball spin rate was marginally larger. It has been shown that the top spin rate negatively correlated with the racquet-face angel at ball impact [7]. The result of this study followed this theory: closed racquet-face angles observed in the Above condition were assumed as the primal factor of the resultant higher ball spin rate. It is interesting to note that off-center, upperside ball impact opened the racquet face just after ball impact; however, the ball launch angle was most likely determined by the initial angle of racquet-face at ball impact, indicating that the opened racquet face typically seen in the Above condition had little effect on resultant ball trajectory. Since the right-handed forehand groundstrokes perform a left-turning motion of the trunk, the ball tends to be launched rightward when players hit the ball in delayed timing. From the result of ball passing position on the net, the ball was delivered more rightward by approximately $30 \mathrm{~cm}$ in the Above condition (Figure 3c). Therefore, it can be assumed that a delayed timing of ball impact most likely induces this type of off-center hitting.

In the Above condition, significantly greater EMG activity for extensor carpi radialis than the Centre condition was observed immediately after ball impact. Figure 2 shows a typical example of the EMG of the ECR and the ulnar/radial deviation angle of the wrist joint of one subject. As radial 
deviation of the wrist joint toward ball impact was commonly observed in all the three conditions, it can be assumed that this motion has a role to accelerate the racquet head forward from the early acceleration to the late acceleration phases (phase I and II). Immediately after phase III, a rapid radial and ulnar deviation of the wrist were observed in the Below and Above conditions, respectively, while there was little change in ulnar/radial deviation in the Centre condition. As the extensor carpi radialis muscle is one of the major agonists of the radius deviation of the wrist, it can be assumed that the ECR is forced to stretch eccentrically in the Above condition.

To our knowledge, this is the first study to investigate the relationships among ball impact location, dynamic racquet behavior, muscle activity and flight behavior of the ball simultaneously during the forehand groundstrokes. We have succeeded in demonstrating the effect of offlongitudinal axis impacts on the above-mentioned variables; however, there were no significant differences in these parameters regarding off-lateral axis impacts (data not shown). This finding is in agreement with previous studies of one-handed backhand groundstrokes [2,3]. However, our finding about the EMG of the ECR contradicts previous findings observed in backhand strokes [2,3]. In these studies, the off-center, lower-side of ball impact induced an eccentric stretch of the wrist extensors. The discrepancy of these results can be explained by mirroring wrist posture between the forehand and backhand strokes while the wrist is pronated during the follow-through phase in both strokes. Since the grip firmness would affect racquet kinematics just after ball impact, the lack of grip firmness data should be noted as the main limitation of this study. Further studies are needed in order to consider the differences in experimental methods, subject tennis skill and motion.

\section{Conclusions}

The representative kinematic of racquet-ball impact were demonstrated in this study, using a high-speed motion capture system $(2000 \mathrm{~Hz})$. It was found that an off-center ball impact occurring above the longitudinal axis of racquet face induces a greater muscular activity in extensor carpi radialis immediately after ball impact, compared to the other ball impact conditions. Furthermore, this type of off-center ball impact likely occurs when players hit the ball in delayed impact timing; as a result, the shot comes to have a lower launch angle and outward launch direction.

Acknowledgments: This study was financially supported by YONEX CO., LTD.

Conflicts of Interest: G Omori and H. Nunome has received research support from YONEX CO., LTD. M. Ikenaga, N. Okuma, H. Nishiyama and S. Chiba are employees of the YONEX CO., LTD. However, the funders had no role in the design of the study; in the collection, analyses, or interpretation of data; in the writing of the manuscript, and in the decision to publish the results.

\section{References}

1. Hennig, E.M.; Rosenbaum, D., Milani, T.L. Transfer of tennis racket vibrations onto the human forearm. Med. Sci. Sports Exerc. 1992, 24, 1134-1140.

2. King, M.A.; Kentel, B.B.; Mitchell, S.R. The effects of ball impact location and grip tightness on the arm, racquet and ball for one-handed tennis backhand groundstrokes. J. Biomech. 2012, 45, 1048-1052. doi:10.1016/j.jbiomech.2011.12.028.

3. King, M.A.; Hau, A.; Blenkinsop, G.M. The effect of ball impact location on racket and forearm joint angle changes for one-handed tennis backhand groundstrokes. J. Sports Sci. 2017, 35, 1231-1238. doi:10.1080/02640414.2016.1211308.

4. Kelley, J.D.; Lombardo, S.J.; Pink, M.; Perry, J.; Giangarra, C.E. Electromyographic and cinematographic analysis of elbow function in tennis players with lateral epicondylitis. Am. J. Sports Med. 1994, 22, 359-363. doi:10.1177/036354659402200311.

5. Hatze, H. Forces and duration of impact, and grip tightness during the tennis stroke. Med. Sci. Sports 1976, 8, 88-95. doi:10.1177/036354659402200311. 
6. Murata, M.; Fujii, N.; Suzuki, Y. Mechanical energy flow of the racquet holding arm in the tennis serve, focusing on the energy form. Jpn. J. Phys. Educ. Health Sport Sci. 2015, 60, 177-195.

7. Kwon, S.; Pfister. R.; Hager, R.L.; Hunter, I.; Seeley, M.K. Influence of Tennis Racquet Kinematics on Ball Topspin Angular Velocity and Accuracy during the Forehand Groundstroke. Sports Sci. Med. 2017, 16, 505-513.

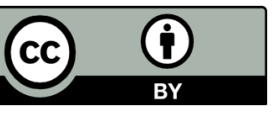

(C) 2020 by the authors. Licensee MDPI, Basel, Switzerland. This article is an open access article distributed under the terms and conditions of the Creative Commons Attribution (CC BY) license (http://creativecommons.org/licenses/by/4.0/). 